\title{
Membrane Protein Profiling of Human Islets of Langerhans Using Several Extraction Methods
}

\author{
Sara F. Hansson • Åsa Henriksson • Lars Johansson • \\ Olle Korsgren • Jan W. Eriksson • Hans Tornqvist • \\ Pia Davidsson
}

Published online: 22 October 2010

(C) Springer Science+Business Media, LLC 2010 total protein extraction, and lipid-based protein immobilization flow cell. Digested protein extracts were analyzed by nanoflow liquid chromatography tandem mass spectrometry. Then the identified proteins were categorized according to cellular location using their gene ontology annotation and by prediction of transmembrane helices in the sequence. This information was used to estimate the amount of membrane proteins identified.

Results By combining the results from all extraction procedures, the total number of membrane proteins identified from the human islets was increased, accentuating that
Electronic supplementary material The online version of this article (doi:10.1007/s12014-010-9060-1) contains supplementary material, which is available to authorized users.

\author{
S. F. Hansson · O. Korsgren \\ Division of Clinical Immunology, Department of Oncology, \\ Radiology and Clinical Immunology, Uppsala University, \\ Uppsala, Sweden \\ O. Korsgren \\ e-mail: olle.korsgren@klinimm.uu.se \\ Å. Henriksson · L. Johansson · J. W. Eriksson • H. Tornqvist · \\ P. Davidsson \\ AstraZeneca R\&D Mölndal, \\ Mölndal, Sweden \\ A. Henriksson \\ e-mail: asa.henriksson@astrazeneca.com \\ L. Johansson \\ e-mail: lars.johansson@radiol.uu.se \\ L. Johansson \\ e-mail: lars.a.johansson@astrazeneca.com \\ J. W. Eriksson \\ e-mail: jan.eriksson@astrazeneca.com \\ H. Tornqvist \\ e-mail: hans.tornqvist@astrazeneca.com
}

\author{
P. Davidsson \\ e-mail: pia.davidsson@astrazeneca.com \\ L. Johansson \\ Division of Radiology, Department of Oncology, Radiology and \\ Clinical Immunology, Uppsala University, \\ Uppsala, Sweden \\ O. Korsgren \\ The Nordic Network for Clinical Islet Transplantation, \\ Gothenburg, Sweden \\ J. W. Eriksson \\ Department of Molecular and Clinical Medicine, Sahlgrenska \\ University Hospital, University of Gothenburg, \\ Gothenburg, Sweden \\ S. F. Hansson $(\bowtie)$ \\ Proteomic Core Facility at University of Gothenburg, \\ P.O. Box 413, 40530 Gothenburg, Sweden \\ e-mail: sara.hansson@gu.se
}


a combination of methods usually gives a higher coverage of the proteome. A total of 1,700 proteins were identified ( $\geq 2$ unique peptides), and 735 of these proteins were annotated as membrane proteins while 360 proteins had at least one predicted transmembrane helix. The extraction method using phase separation with Triton X-114 yielded both the highest number and the highest proportion of membrane proteins.

Conclusion This study gave an enhanced characterization of the human islet membrane proteome which may contribute to a better understanding of islet biology.

Keywords Human islets of Langerhans · Proteomics . Membrane protein extraction - Mass spectrometry . SDS-PAGE · FTICR MS/MS

\section{Introduction}

The islets of Langerhans, constituting $1 \%$ to $2 \%$ of the pancreas mass, compose the endocrine part of the pancreas. The main cell types in the islets are the insulin and amylin producing beta-cells as well as the glucagon producing alpha-cells, while somatostatin producing delta-cells and pancreatic polypeptide producing cells are less abundant $[1$, 2]. Hormones released from these cells serve complex, synergistic or antagonistic, roles maintaining the homeostasis of plasma glucose levels in the body under normal conditions. In diabetes mellitus, there is an absolute (type 1 diabetes mellitus) or relative insulin deficit (type 2 diabetes mellitus) [3], leading to chronic hyperglycemia and other metabolic perturbations.

Pancreatic beta-cells are destroyed in patients with type 1 diabetes as a result of selective autoimmune destruction [4]. In patients with type 2 diabetes, insulin resistance is usually combined with a progressive loss of beta-cell function and mass occurring over time [5]. Insulin resistance in peripheral tissues challenges the beta-cells to produce and secrete increased amounts of insulin to maintain normoglycemia. The insulin resistance and the gradual loss of beta-cell function finally lead to insufficient insulin release and chronic hyperglycemia. The prolonged exposure of beta-cells to high glucose levels (glucotoxicity) further reduces the capacity of beta-cells to secrete insulin, thus creating a vicious circle [6]. The mechanism causing the loss of beta-cell function and mass in type 2 diabetes is still debated [3].

In order to better understand the pathophysiology of both type 1 and type 2 diabetes mellitus, an improved characterization of the islets of Langerhans and their response to glucotoxicity is essential. With these aims, several proteomic studies have been conducted $([7,8]$ for recent reviews). The majority of previous proteomic studies have been performed on cultured insulinoma cells [9-13] or mouse islets [14-18]. However, these model systems might not reflect the human systems $[19,20]$. Two proteomic studies have previously been performed on human pancreatic islets. The first aimed at creating a twodimensional gel electrophoresis (2-DE) reference map of human islets and generated 66 unique protein identities [21]. Then, Metz et al. [22] performed a global characterization of total extracts from human islets of Langerhans using two-dimensional liquid chromatography (LC) tandem mass spectrometry (MS/MS). Our proteomic study is focused on characterizing the membrane proteome of human islets. Membrane proteins play a critical role in cellular function and currently account for about $70 \%$ of all known pharmaceutical drug targets [23]. A better definition of the membrane proteome of human islets would add important knowledge for our endeavors in finding and better understand new targets in human islets, which may lead to new therapies aiming at preserving or even increasing the number of beta-cells. It may also help in discovery of novel biomarkers, both for measurement of circulating levels and for imaging approaches, which can reflect beta-cell mass and function.

In the present study, we have used isolated human pancreatic islets to characterize the membrane proteome. For an improved membrane islet protein profiling, five different extraction procedures have been used and compared followed by identification of membrane proteins by nanoflow reversed phase LC coupled with Fourier transform ion cyclotron resonance (FTICR) MS/MS.

\section{Materials and Methods}

\section{Human Islets of Langerhans}

Pancreatic tissues that were not used for transplantation were obtained from multiorgan donors with informed consent either from the organ donor registry or from relatives. Approval for the conduct of this study was obtained from the local ethics committee. Human pancreatic islets were isolated, as described previously [24], at Uppsala University, using intraductal collagenase perfusion, automated digestion filtration, islet continuous gradient purification, and subsequent islet culture for 6 days in CMRL 1066 (AppliChem, GmbH, Darmstadt, Germany) supplemented with $50 \mu \mathrm{g} / \mathrm{mL}$ gentamycin and $0.25 \mu \mathrm{g} / \mathrm{mL}$ fungizone (GIBCO BRL) at $37^{\circ} \mathrm{C}(5 \%$ carbon dioxide). Islet yield and purity were determined in a standardized procedure using a digital analysis system (Cellimag, Uppsala, Sweden). All analyzes were performed on snap frozen islet tissue ( $\geq 90 \%$ pure) from the same donor. The islets were kept at $-80^{\circ} \mathrm{C}$ until analysis. 


\section{Protein Extraction Procedures}

Five different approaches were tested for solubilization of membrane proteins from human pancreatic islets (Fig. 1), including total protein extraction, phase separation using Triton X-114, plasma membrane protein extraction, cell surface protein biotinylation, and lipid-based protein immobilization (LPI).

Total Protein Extraction Human pancreatic islets (45 mg, corresponding to 20,000 islets equivalents (IEQ)) were dissolved in lysis buffer containing $7 \mathrm{M}$ urea, $2 \mathrm{M}$ thiourea, $30 \mathrm{mM}$ tris(hydroxymethyl)aminomethane (Tris), 4\% 3-[(3cholamidopropyl) dimethylammonio]-1-propanesulfonate, and $1 \%(v / v)$ protease inhibitor cocktail Set III (Calbiochem, Merck Chemicals, Nottingham, UK). The cells were sonicated using a Soniprep 150 Ultrasonic Disintegrator (MSE Limited, London, UK) on ice/water bath for four 10-s bursts with 20-s intervals between each burst. Cell debris was removed by centrifugation at $15,000 \times g$ for $30 \mathrm{~min}$ at $4^{\circ} \mathrm{C}$, and the supernatant was transferred to new vials. A fraction of the protein extract (100 $\mu \mathrm{g}$ protein concentration) was purified and concentrated using the ProteoExtract kit (Calbiochem, Merck Chemicals) dissolved in NuPAGE lithium dodecyl sulfate (LDS) sample buffer (Invitrogen Limited, Paisley, UK) containing $50 \mathrm{mM}$ 1,4-dithiothreitol (DTT) and stored at $-80^{\circ} \mathrm{C}$ pending analysis.

Phase Separation Using Triton X-114 Proteins from human pancreatic islets (57 mg, corresponding to 25,000 IEQ) were extracted using the ReadyPrep extraction kit, membrane 1 (Bio-Rad, Hercules, CA, USA), which is based on the method described by Bordier [25], according to instructions from the manufacturer. Briefly, islets were sonicated in a Triton X-114 buffer containing protease inhibitors using a Soniprep 150 Ultrasonic Disintegrator (MSE Limited) on ice/water bath for four 10-s bursts with 20-s intervals between each burst. Temperature-dependent phase partitioning and centrifugation at $13,000 \times g$ for $10 \mathrm{~min}$ at room temperature (RT) generated membrane proteins in a hydrophobic phase (Triton X-114) and an insoluble pellet. A fraction of each protein extract $(25 \mu \mathrm{g}$ protein concentration) was purified and concentrated using the ProteoExtract kit (Calbiochem) dissolved in LDS sample buffer (Invitrogen) containing $50 \mathrm{mM}$ DTT and stored at $-80^{\circ} \mathrm{C}$ pending analysis.

Plasma Membrane Protein Extraction Kit Using Lectin Binding of Microsomes Human islets (190 mg, corresponding to 80,000 IEQ) were treated with the Qproteome plasma membrane protein kit (Qiagen Group, Valencia, CA, USA) according to instructions from the manufacturer. Briefly, the islet cells were mechanically disrupted, in the provided lysis buffer containing protease inhibitors, by injecting and rejecting the lysate for 20 times in a disposable needle and syringe. Then, the lysate was centrifuged at $12,000 \times \mathrm{g}$ for $20 \mathrm{~min}$ at $4^{\circ} \mathrm{C}$, and the pellet was ejected. Plasma membrane microsomes were extracted from the supernatant by addition of magnetic beads coated with lectins (biotinylated wheat germ agglutinin, concanavalin A, and Lens culinaris). After washing, plasma membrane vesicles were eluted under native conditions. The protein extract ( $25 \mu \mathrm{g}$ protein concentration) was purified and concentrated using the ProteoExtract kit
Fig. 1 Schematic presentation of the workflow for the analysis of proteins from human pancreatic islets in this study

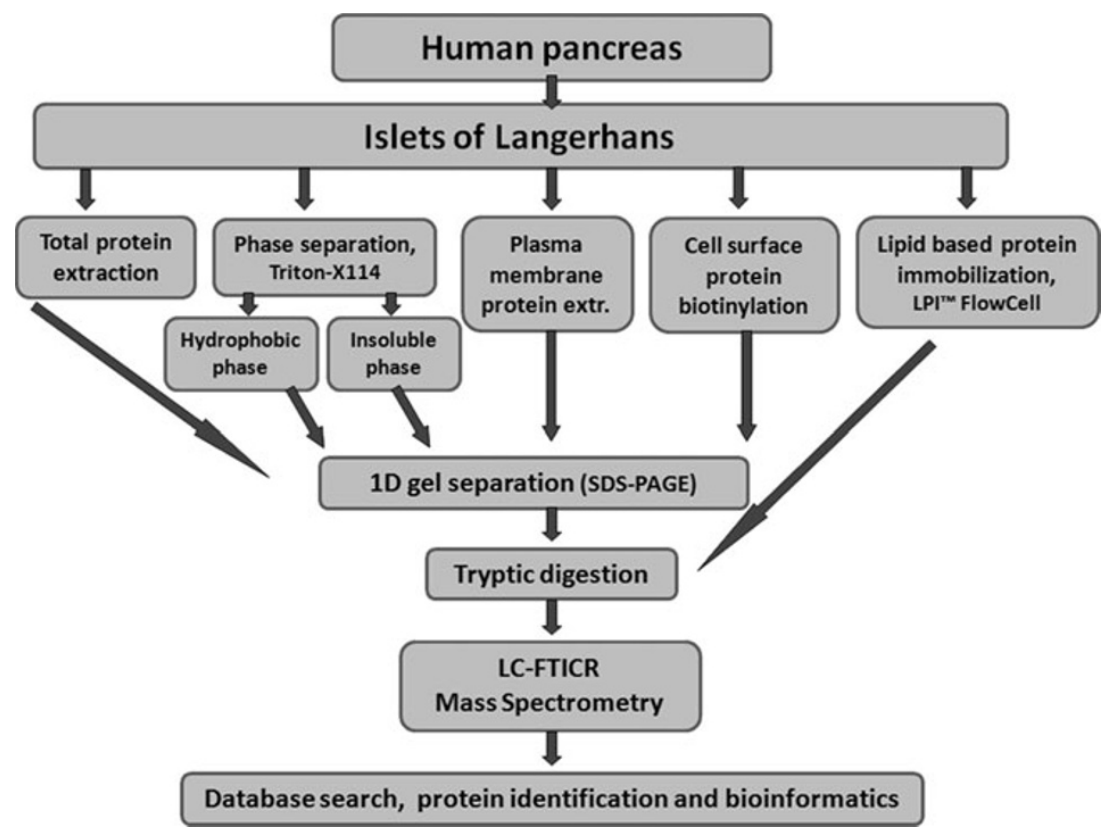


(Calbiochem) dissolved in LDS sample buffer (Invitrogen) and stored at $-80^{\circ} \mathrm{C}$ pending analysis.

Cell Surface Protein Biotinylation Human islets (127 mg, corresponding to 55,000 IEQ) were treated with the Cell Surface Protein Isolation Kit (Pierce, Rockford, IL, USA) according to instructions from the manufacturer. Cell surface proteins on intact islet cells were labeled with EZLink Sulfo-NHS-SS-Biotin. Cells were lysed with a mild detergent containing protease inhibitors using a Soniprep 150 ultrasonic disintegrator (MSE Limited) on ice/water bath for five 10-s bursts with 30 -s intervals between each burst. The labeled proteins were isolated using a NeutrAvidin agarose spin column, and the proteins were eluted after incubation with $50 \mathrm{mM}$ DTT in LDS sample buffer (Invitrogen) and stored at $-80^{\circ} \mathrm{C}$ pending analysis.

Lipid-Based Protein Immobilization Flow Cell Crude membrane extracts were prepared from human pancreatic islets (68 mg, corresponding to 30,000 IEQ) by addition of $10 \mathrm{mM}$ $\mathrm{NaHCO}_{3}$ and Dounce homogenization followed by centrifugation at $10,000 \times \mathrm{g}$, at $4^{\circ} \mathrm{C}$ for $10 \mathrm{~min}$. Addition of $100 \mathrm{mM} \mathrm{Na} \mathrm{CO}_{3}$ to the supernatant containing the crude membranes removed peripheral membrane proteins, and the solution was centrifuged at $50,000 \times \mathrm{g}$, at $4^{\circ} \mathrm{C}$ for $90 \mathrm{~min}$. The pelleted crude membrane extracts were diluted in buffer (10 mM Tris- $\mathrm{HCl}, 300 \mathrm{mM} \mathrm{NaCl}, \mathrm{pH} 8)$ and tip sonicated to form proteoliposomes and then immobilized on the surface of the LPI flow cell (Nanoxis, Gothenburg, Sweden), followed by tryptic digestion of membrane proteins in the proteoliposomes and elution of peptides from the flow cell. The peptide extract was dried in a vacuum centrifuge (DNA 120 SpeedVac, ThermoSavant) and dissolved in $0.2 \%$ formic acid prior to LC-MS/MS analysis.

Prior to freezing, a small aliquot from each extract was removed for determination of protein concentration. The aliquots were treated with the ProteoExtract protein precipitation kit (Calbiochem, Merck Chemicals) to remove chemicals that could interfere with the protein assay. Proteins were solubilized in phosphate buffered saline, and the protein concentration was determined using bicinchoninic acid protein assay kit (Pierce).

\section{SDS-PAGE}

Extracted proteins were separated by sodium dodecyl sulfate-polyacrylamide gel electrophoresis (SDS-PAGE). The extracts dissolved in NuPAGE LDS sample buffer (Invitrogen) containing $50 \mathrm{mM}$ DTT were thawed, heated to $56^{\circ} \mathrm{C}$ for $10 \mathrm{~min}$, and separated on $10 \%$ Bis-Tris NuPAGE gels, $1 \mathrm{~mm}$, ten wells (Invitrogen) with $1 \times$ MES buffer (Invitrogen, $50 \mathrm{mM}$ 2-( $N$-morpholino)ethanesul- fonic, $50 \mathrm{mM}$ Tris base, $1 \mathrm{mM}$ EDTA, $3.5 \mathrm{mM}$ SDS at $\mathrm{pH} 7.3$ ) at $200 \mathrm{~V}$ for $37 \mathrm{~min}$. The gels were stained using SimplyBlue Coomassie SafeStain (Invitrogen).

\section{In-Gel Protein Digestion}

Each SDS-PAGE lane was excised and cut in 15 pieces and subjected to in gel tryptic digestion as previously described by Shevchenko et al. [26], with some minor modifications. Briefly, the gel pieces were destained by washing twice in $25 \mathrm{mM} \mathrm{NH}_{4} \mathrm{HCO}_{3}$ in $50 \% \mathrm{CH}_{3} \mathrm{CN}$. Gel pieces were dried in a vacuum centrifuge and incubated with $10 \mathrm{mM}$ DTT in $25 \mathrm{mM} \mathrm{NH}_{4} \mathrm{HCO}_{3}$ for $40 \mathrm{~min}$ at $56^{\circ} \mathrm{C}$ followed by a dark incubation with $55 \mathrm{mM}$ iodoacetic acid in $25 \mathrm{mM}$ $\mathrm{NH}_{4} \mathrm{HCO}_{3}$ for $30 \mathrm{~min}$ at RT. Then, the gel pieces were washed twice in $25 \mathrm{mM} \mathrm{NH}_{4} \mathrm{HCO}_{3}$ in $50 \% \mathrm{CH}_{3} \mathrm{CN}$ and once in $25 \mathrm{mM} \mathrm{NH}_{4} \mathrm{HCO}_{3}$ in $50 \% \mathrm{CH}_{3} \mathrm{OH}$ and dried in the vacuum centrifuge. Digestion buffer $\left(50 \mathrm{mM} \mathrm{NH}_{4} \mathrm{HCO}_{3}\right.$, $10 \mathrm{ng} / \mu \mathrm{L}$ trypsin) was added to the dried gel pieces followed by incubation at $37^{\circ} \mathrm{C}$ overnight. Peptides were extracted in $50 \% \mathrm{CH}_{3} \mathrm{CN}$ containing $1 \% \mathrm{CH}_{3} \mathrm{COOH}$ twice, and the supernatants were pooled and evaporated to dryness in the vacuum centrifuge. Prior to MS analysis, the peptides were reconstituted in $0.1 \% \mathrm{HCOOH}$.

\section{Nanoflow LC-MS/MS FTICR}

Sample injections were made with an HTC-PAL autosampler (CTC Analytics AG, Zwingen, Switzerland) connected to an Agilent 1,100 binary pump (Agilent Technologies, Palo Alto, CA, USA). The peptides were trapped on a precolumn ( $45 \times$ $0.075 \mathrm{~mm}$ i.d.) and separated on a reversed phase column, $200 \times 0.050 \mathrm{~mm}$. Both columns are packed in-house with $3 \mu \mathrm{m}$ Reprosil-Pur C18-AQ particles. The flow through the analytical column was reduced by a split to approximately $100 \mathrm{~nL} /$ min. A 40 -min gradient $10-50 \% \mathrm{CH}_{3} \mathrm{CN}$ in $0.2 \% \mathrm{COOH}$ was used for separation of the peptides. For more details, see [27].

The nanoflow LC-MS/MS were performed on a hybrid linear ion-trap FTICR mass spectrometer equipped with a 7-T ICR magnet (LTQ-FT, Thermo Electron, Bremen, Germany). The spectrometer was operated in datadependent mode, automatically switching to MS/MS mode. MS-spectra were acquired in the FTICR, while MS/MSspectra were acquired in the LTQ-trap. For each scan of FTICR, the six most intense, doubly or triply charged, ions were sequentially fragmented in the linear trap by collisioninduced dissociation. Between scans, an exclusion time of $6 \mathrm{~s}$ for the same ion species was selected.

Database Search and Data Analysis

All MS/MS samples were analyzed using Mascot v 2.2.1 (Matrix Science, London, UK) and X!Tandem (www. 
thegpm.org; version 2007.01.01.1). X!Tandem was set up to search a subset of the UniProtKB/Swiss-Prot database (http://www.expasy.ch/sprot/) also assuming trypsin. Mascot was set up to search the UniProtKB/Swiss-Prot 55.3 database (selected for Homo sapiens, 19372 entries).

Mascot and X!Tandem were searched with a fragment ion mass tolerance of $0.50 \mathrm{Da}$ and a parent ion tolerance of $5.0 \mathrm{ppm}$. Iodoacetamide derivative of cysteine (for total protein extraction, hydrophobic phase, insoluble phase, plasma membrane protein extraction, and cell surface protein biotinylation samples) was specified in Mascot and X!Tandem as a fixed modification. Oxidation of methionine was specified in Mascot and $\mathrm{X}$ !Tandem as a variable modification.

Criteria for Protein Identification Scaffold (version Scaffold_2_05_02, Proteome Software Inc., Portland, OR, USA) was used to validate MS/MS-based peptide and protein identifications. Peptide identifications were accepted if they could be established at greater than $95.0 \%$ probability as specified by the PeptideProphet algorithm [28]. Protein identifications were accepted if they could be established at greater than $99.0 \%$ probability and contained at least two identified unique peptides (i.e., identified peptide sequences that are unique to an individual parent protein sequence). Protein probabilities were assigned by the ProteinProphet algorithm [29]. Proteins that contained similar peptides and could not be differentiated based on MS/MS analysis alone were grouped to satisfy the principle of parsimony.

When the rule for protein identification was applied $(\geq 2$ unique peptides at $\geq 95 \%$ level of confidence), the false discovery rate of protein identifications was $0.64 \%$. Furthermore, according to the ProteinProphet algorithm [29], no identified protein had a probability of less than $99.8 \%$.

The GO TermMapper (http:/go.princeton.edu/cgi-bin/ GOTermMapper) was used to annotate the cellular location of proteins.

Prediction of transmembrane helices $(\mathrm{TMH})$ in proteins was performed by CBS prediction servers (www.cbs.dtu. $\mathrm{dk} /$ services/TMHMM/).

Proteins identified in digests from "blank" gel pieces, not containing any islet protein extracts, were considered contaminants and removed from the list of proteins; these proteins all correspond to different keratins (e.g., keratin, type II cytoskeletal 1, 5, 6A, 7, 8, and 9; keratin, type I cytoskeletal 10,13,14,16, 18, and 19 as well as keratin, type II cytoskeletal two epidermal).

\section{Results and Discussion}

This study focuses on characterization of the membrane fraction of human islets of Langerhans and especially on proteins located at the plasma membrane and cell surface since most drug targets have been suggested with this cellular location [30]. For an improved membrane protein profiling of the islets, five distinct protein extraction approaches were used including total extraction, phase separation using triton $\mathrm{X}-114$ (enriching membrane proteins in a hydrophobic phase and an insoluble pellet), a plasma membrane protein kit, cell surface protein biotinylation, and LPI flow cell, followed by LC-MS/MS analysis (Fig. 1).

In total, 1,700 proteins from pancreatic islets were identified in this study, and 930 of these proteins were confirmed by at least two different extraction methods (Supplementary Table 1).

The identified proteins were assigned to cellular location using gene ontology (GO) annotations, and the presence of $\mathrm{TMH}$ in their sequence was predicted in order to analyze the performance of the different extraction methods (Fig. 2; Table 1). The total amount of unique peptides and proteins identified by each individual protein extraction procedure showed that the highest number of proteins $(1,136)$ was detected when analyzing total protein extracts of the islets (Table 1). One reason is that the total extract was applied in fourfold total protein concentration compared to the other methods. Since the same starting amount of islet tissue generally gave a fourfold protein yield when using total protein extraction compared to the membrane protein enriching methods, this ratio was kept in the mass spectrometric analysis. If the total extraction procedure could identify a similar amount of membrane proteins as the membrane enriching methods, this would be an easy and straightforward method to use. Thus, these methods were further compared. By combining all unique proteins identified after each membrane enriching extraction procedure, a total of 1,348 proteins were identified, and 564 of these proteins were not identified using only total protein extraction (Fig. 3). As anticipated, a high proportion $(61.2 \%)$ of membrane proteins was present among the 564 proteins exclusively identified by the membrane enriching methods. Furthermore, only $18.5 \%$ of the 342 proteins exclusively identified in the total extract were membrane proteins. These results highlight that in the analysis of proteins in complex biological samples, like the islets of Langerhans, sample preparation and pre-fractionation can increase the amount of identified proteins with specific properties, which are favored by the sample preparation method. By combining results from several protein extraction procedures, a more complete picture of the proteome can be created [31].

However, it is not always possible to combine results from different proteomic procedures, e.g., due to limited amounts of biological sample, costs, and/or time considerations. Thus, the most suitable method has often to be selected. The hydrophobic phase extraction yielded the highest proportion of proteins annotated to membrane, 


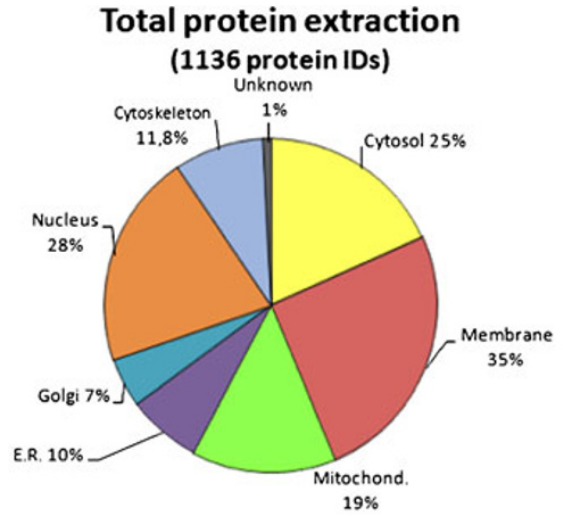

Plasma membrane prot. extr. (386 protein IDs)

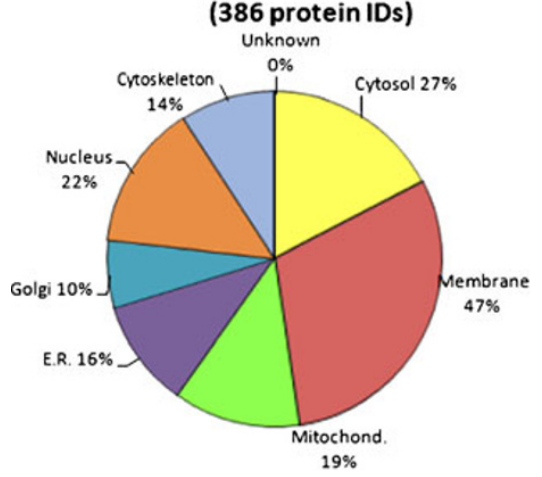

Fig. 2 Extraction efficiency of proteins from different cellular locations. Pie chart showing cellular location of identified proteins after the different protein extraction methods. The percentages were determined using the GO TermMapper, cellular component ontology.

plasma membrane, and cell surface, compared with all other extraction methods (Table 1). Furthermore, the presence of TMH in the identified protein sequences was predicted, showing that the LPI flow cell method extracted the highest proportion (42\%) of these TMH proteins (Table 1).

The four extraction methods with the highest proportion of membrane proteins (that is, the hydrophobic phase of Triton X-114 extraction, plasma membrane protein extraction, cell surface protein biotinylation, and LPI flow cell) were further compared by determining the number of protein identities overlapping between the methods. It was evident that the hydrophobic phase yielded the highest number of proteins that were only identified by this method, both when comparing the number of plasma membrane proteins (Fig. 4a) as well as TMH proteins (Fig. 4b). Unexpectedly, the methods designated to enrich plasma membrane proteins as well as cell surface proteins extracted relatively few uniquely identified plasma membrane proteins (Fig. 4a). The plasma membrane extraction kit is based on selective lectin binding of the carbohydrates on plasma membrane protein vesicles. This selective interaction might have been reduced by any interfering protein or if a proportion of the plasma membrane vesicles

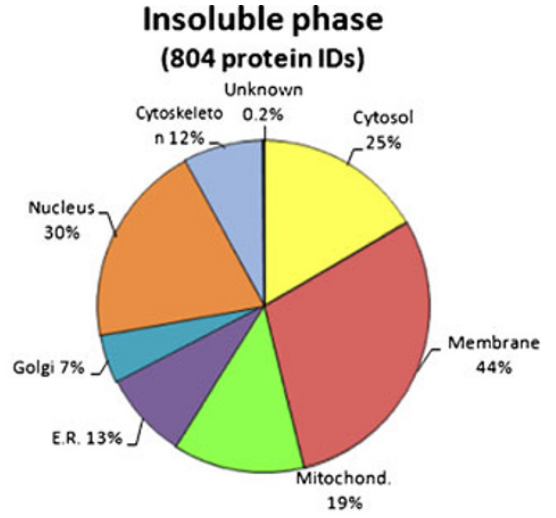

LPI Flow cell

(143 protein IDs)

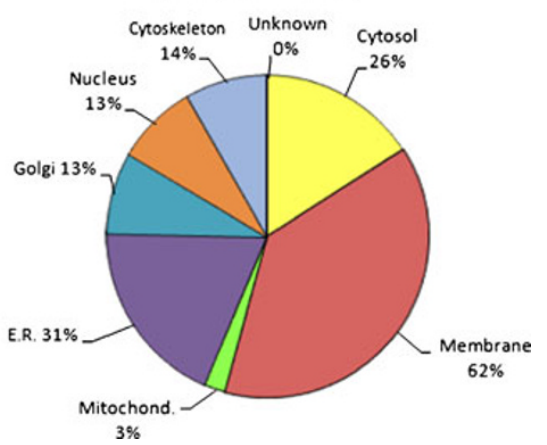

Protein identification, at $>99 \%$, required at least two unique peptides, at $\geq 95 \%$ confidence level, detected by LC-FTICR MS/MS, searched against the human Swiss-Prot database using MASCOT and X! Tandem

were turned inside-out in the lysis procedure (i.e., leaving the carbohydrate moiety of the plasma membrane proteins hidden inside of the vesicle). In addition, high abundant soluble proteins might have been trapped inside the vesicles and extracted together with the plasma membrane proteins, explaining the low enrichment of plasma membrane proteins.

The limited success of the cell surface biotinylation kit could be explained since labeling may have been prevented by steric hindrance, lack of primary amines in the protein sequences extending from the cells, and/or a minimal sequence with extracellular exposure. Furthermore, this kit was developed for the analysis of cell cultures and not for tissue samples; thus, any lysis of cells etc. may cause intracellular proteins to be available for biotinylation, resulting in reduced proportion of membrane proteins in this extract.

In this study, the only extraction procedure that did not involve a gel separation step prior to LC-MS/MS was the LPI flow cell method. This method yielded the highest percentage of TMH proteins and the second highest percentage of membrane proteins (Table 1). However, due to the total amount of proteins identified by this method 
being quite low, a low coverage of the islet membrane proteome still was given. The low protein yield of this method could at least partly be explained by the fact that this extract was run in a single LC-MS/MS run after elution of peptides from the flow cell, while all the other extraction methods involved a protein separation step on SDS-PAGE resulting in 15 fractions, each digested and analyzed separately by LC-MS/MS. In order to increase the yield of the peptide extract eluted from the LPI flow cell in future analyses, a second separation step on peptide level could be added.

Several characteristic hormones of the islet of Langerhans were detected in this study including glucagon, insulin, islet amyloid polypeptide, and somatostatin. These hormones are secreted molecules and are best represented in the total extraction method (Supplementary Table 1). These results emphasize that soluble proteins are unfavored by the membrane protein enriching methods.

Furthermore, 61 proteins with receptor function were identified (Table 2). The hydrophobic extraction method yielded most of the identified receptors (52 proteins, $85 \%$ ). By combining receptors identified in the hydrophobic phase and the insoluble phase (since they are different phases of the same Triton X-114 extraction), $93 \%$ of the receptor proteins, identified in this study, are covered. Generally, the highest amount of unique peptides and the highest sequence coverage of the receptors were also identified by the hydrophobic phase method (Table 2).

A detailed description of these receptors is beyond the scope of this article; however, a few receptor classes, including integrins and $\mathrm{CD}$ proteins, merit commenting due to their potential involvement in beta-cell destruction or the pathogenesis of diabetes. Integrins are integral receptors, composed of an alpha and beta chain, that mediate attachment between a cell and the tissues surrounding it, which may be other cells or the extracellular matrix (ECM). They also play a role in cell signaling and thereby define cellular shape, mobility, and regulate the cell cycle. Integrins also transduce information from the ECM to the cell as well as reveal the status of the cell to the outside, allowing rapid and flexible responses to changes in the environment [32]. There are many types of integrins, and many cells have multiple types on their surface. Integrins can bind components such as fibronectin, vitronectin, collagen, and laminin. Furthermore, increased levels of several integrins have been found in microvessels isolated from patients with diabetes mellitus [33, 34].

The $\mathrm{CD}$ proteins are members of the transmembrane four superfamily. Most of these members are cell surface glycoproteins that are characterized by the presence of four hydrophobic domains. The proteins mediate signal transduction events that play a role in the regulation of cell development, activation, growth, and motility [35]. It has 


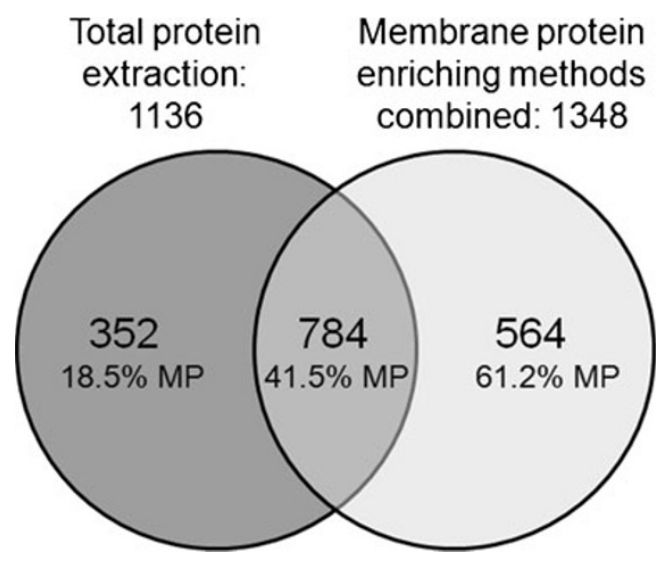

Fig. 3 Venn diagram comparing the numbers and overlap of unique proteins from human pancreatic islets identified after total protein extraction and when all membrane protein enriching extraction methods were combined. The percentages of membrane proteins $(M P)$ are also given. Protein identification, at $>99 \%$, requiring at least two unique peptides, at $\geq 95 \%$ confidence level, detected by LCFTICR MS/MS, searched against the human Swiss-Prot database using MASCOT and X!Tandem

been indicated that the progression of islet destruction in type 2 diabetes islets may be the result of specific changes in pathway activation of signaling related to proliferation and regeneration [36]. CD proteins are known to form complexes with integrins to regulate cell motility in human pancreatic adenocarcinoma [37]. Furthermore, the TSPAN8 gene belonging to the transmembrane four superfamily has been suggested to contain a risk loci for type 2 diabetes making it possible that variation in this protein may biologically influences pancreatic beta-cell function [38].

The successful identification of these receptor proteins indicates that hydrophobic phase extraction is a good method for further studies of these membrane proteins with implications in beta-cell dysfunction or the diabetic pathology. However, as the ATP-binding cassette transporter sub-family $\mathrm{C}$ member eight protein exemplifies, it is likely that the detergent Triton X-114 is unable to solubilize this protein sufficiently, with its six transmembrane helices, and therefore, the protein ended up in the insoluble phase (Table 2). Nevertheless, some highly hydrophobic proteins, e.g., neutral amino acid transporter $\mathrm{B}(0)$, with nine transmembrane helices, could still be identified in the hydrophobic phase of Triton X-114 extracts.

Even so, this study failed to identify some receptors with important functions in the islet cells, for example, the insulin receptor, somatostatin receptor [39], and epidermal growth factor receptor [40]. One explanation can be that these receptors are relatively low abundant and due to the complex mixture of proteins in a biological sample, which has a vast dynamic range of protein amounts [41], the analytic ability of mass spectrometry still makes it hard to detect the low abundant proteins. For detection of very low
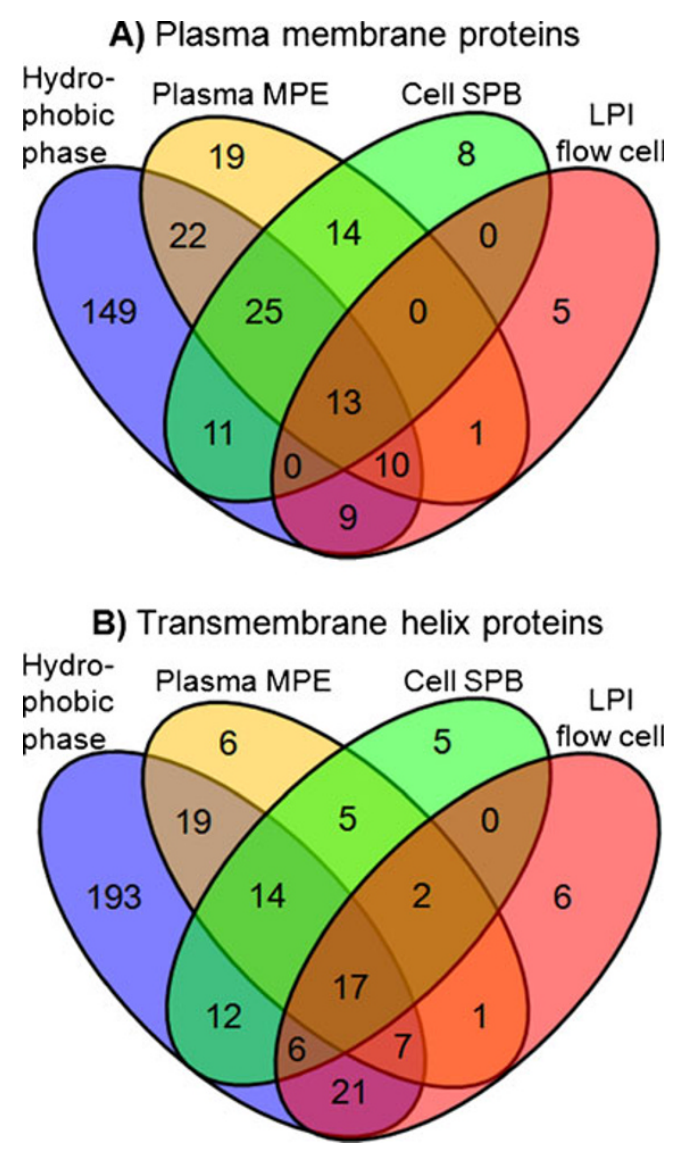

Fig. 4 Venn diagram comparing the numbers and overlap of proteins identified after different membrane protein extractions of human pancreatic islets. a The number of proteins identified as plasma membrane proteins according to gene ontology annotation. b The number of proteins predicted with at least one transmembrane helix in their protein sequence. Protein identification, at $>99 \%$, required at least two unique peptides, at $\geq 95 \%$ confidence level, detected by LCFTICR MS/MS, searched against the human Swiss-Prot database using MASCOT and X!Tandem. Hydrophoboc phase the hydrophobic phase of Triton X-114 phase separation, Plasma MPE plasma membrane protein extraction, Cell $S P B$ cell surface protein biotinylation, LPI flow cell lipid-based protein immobilization flow cell

abundant proteins of the cells, more focused techniques, for example, immunoprecipitation followed by MS/MS identification or antibody based detection methods, are required. A recent study [42] has utilized high throughput immunohistochemistry on tissue microarrays for detection of proteins expressed in pancreatic islets. The Swedish Human Protein Altas [43] (www.proteinatlas.org) was searched for proteins with a differential expression in the endocrine islets compared with the surrounding exocrine pancreatic tissue. They identified five novel endocrine markers with expression in islet cells, both in pancreatic tissues and in vitro cultured islets including, beta-2-microglobulin, glyceraldehyde-3-phosphate dehydrogenase, SUN domaincontaining protein 3 (SUNC1), tetraspanin-7, and ubiquitin carboxyl-terminal hydrolase isozyme L1. These suggested 


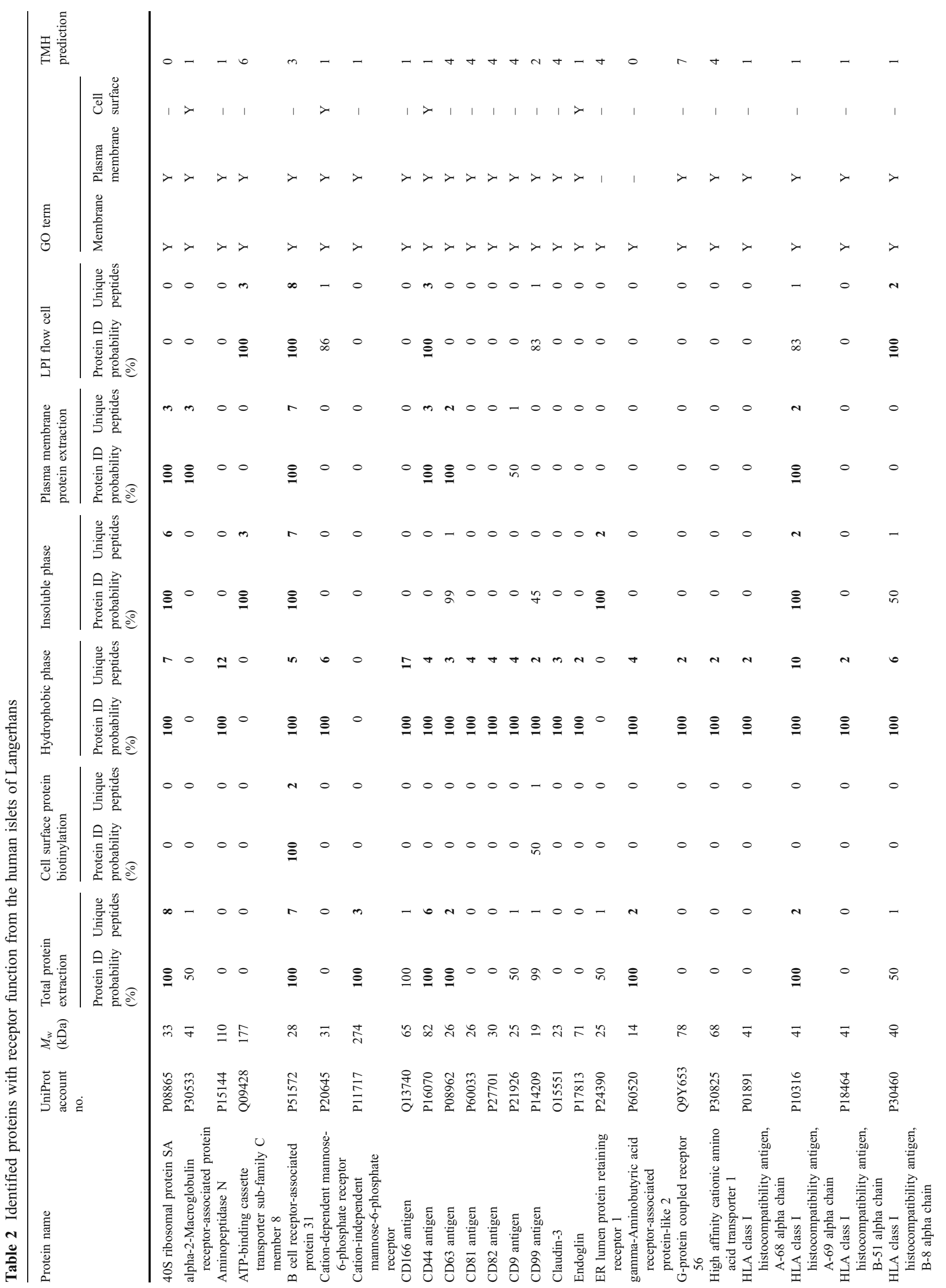




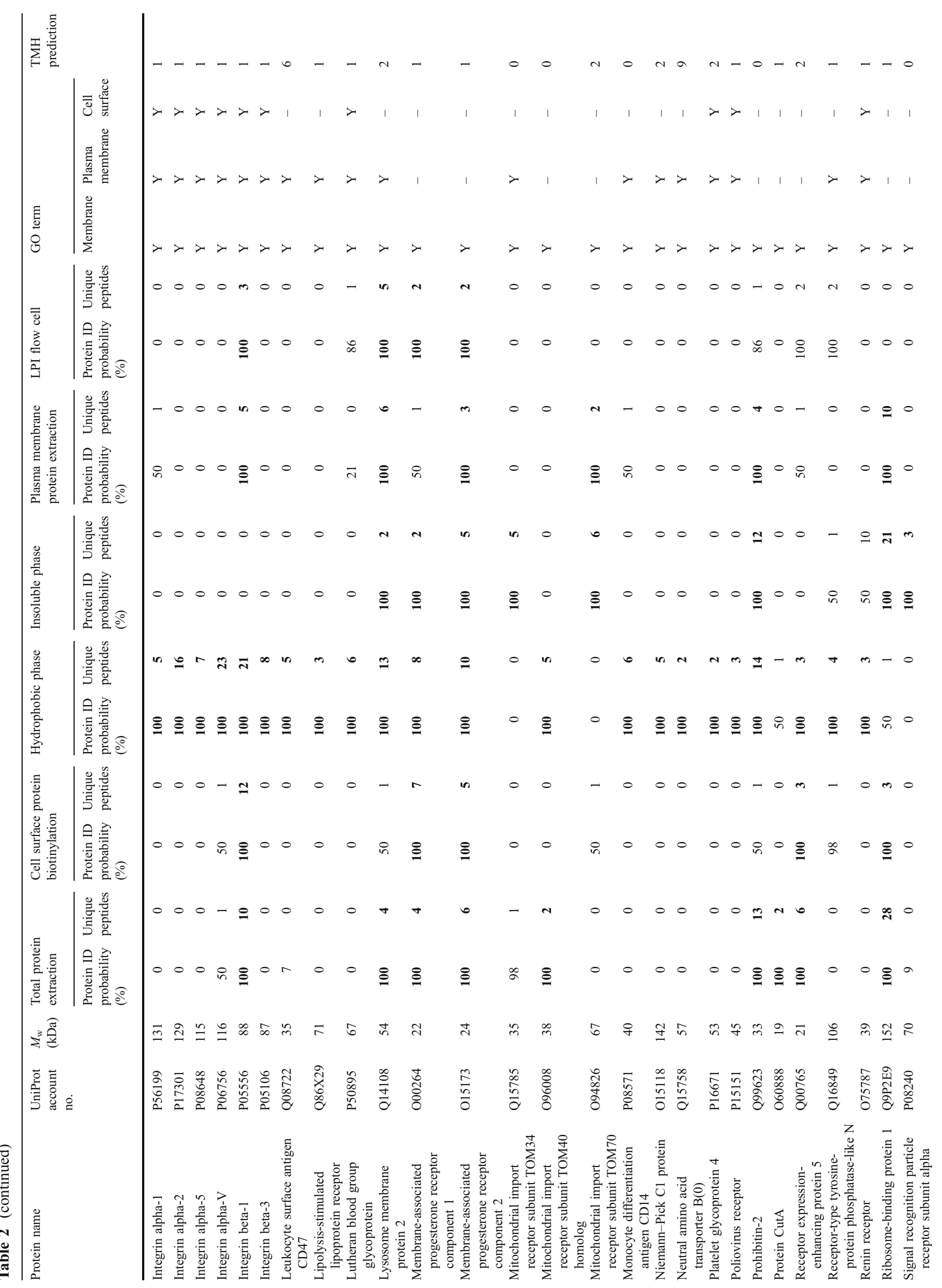




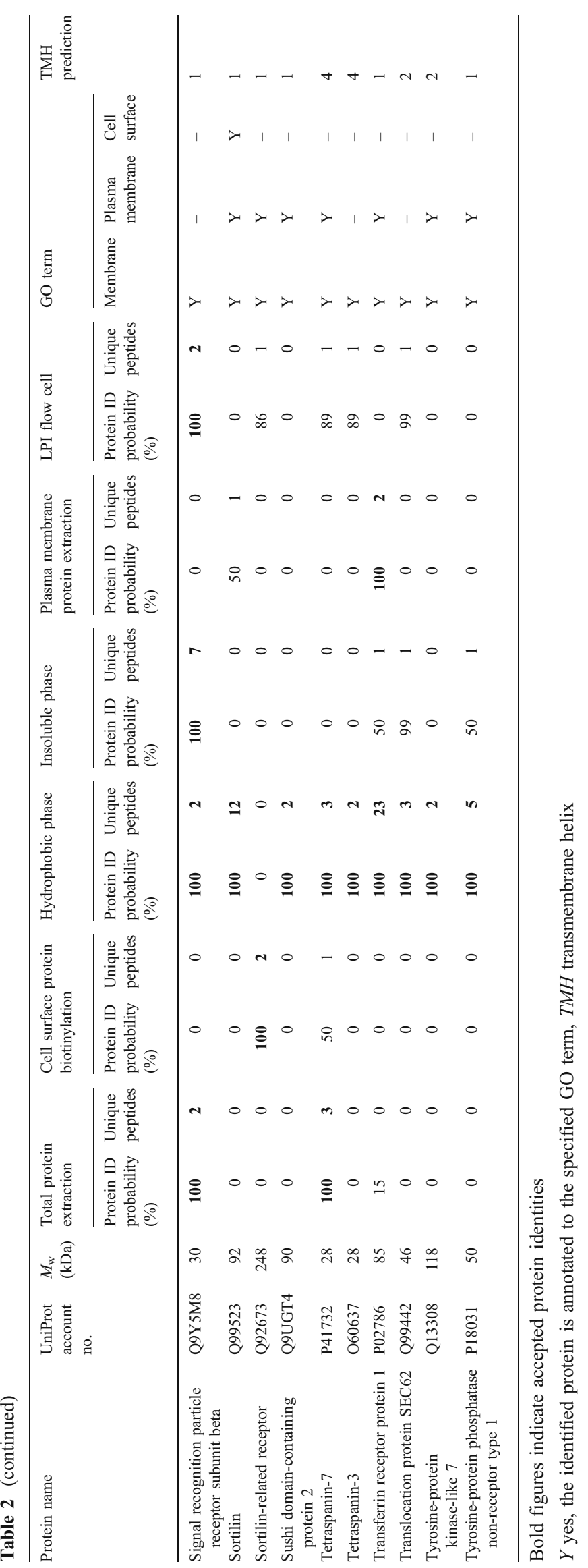


endocrine markers were also identified in our study (Supplementary Table 1), with exception of SUNC1, which may be explained by that SUNC1 had a significantly lower protein level in isolated islets [42], used in our study, compared with that of islets in pancreatic tissues.

The two previous proteomic studies of isolated human pancreatic islets have both characterized total protein extracts. Ahmed et al. [21] used 2-DE and MALDI-TOF-MS and detected 744 protein spots in the gels, 130 of these proteins were identified, and they corresponded to 66 unique proteins. All these proteins, with exception of the glutamate dehydrogenase 2 (mitochondrial), were confirmed in our study. The study by Metz et al. [22] used two-dimensional LC separations coupled with ion-trap MS/MS for a global proteomic characterization of human islets and identified 3,365 proteins ( $\geq 2$ unique peptides) against the human International Protein Index (IPI) database [44]. In our study, focusing on the membrane proteome, the MS/MS data were searched against the human UniProtKB/Swiss-Prot (Sprot) curated protein sequence database, providing a high level of annotation, a minimal level of redundancy, and a high level of integration with other databases. The present study only accepted peptide and protein identifications based on relatively strict criteria of the Prophet algorithms [28, 29]. The use of different experimental procedures and search criteria is a likely explanation for the different number of identified proteins in these studies. Nevertheless, when the protein identities from this study were converted to IPI entries, 1,169 proteins overlapped with the identities in the study by Metz and coworkers [44], while 531 proteins were uniquely identified in our study. Among the proteins identified only in this study, 269 were membrane proteins and 156 contained transmembrane helices. Thus, our study confirms some previous findings and adds further proteins to the proteomic profile of human islets of Langerhans.

Most previous diabetes-related proteomic studies have been performed on pancreatic beta-cell lines, especially mouse insulinoma, MIN-6 cells [10], and rat insulinoma, INS- 1 cell clones $[9,11,12]$ as surrogates for primary $\beta$ cells because they are more abundant and homogenous. However, their expression profile can be significantly different from that of primary $\beta$-cells [19]. On the other hand, proteomic studies on human islets are influenced by the variability introduced by individual changes and by the less controlled conditions that can be applied for the procurement of tissues from humans, relative to cell lines and to laboratory animals. Thus, the advances in producing a reverted immortalized human beta-cell line RNATK-15 [45] might be a valuable source for future investigations in the pathology of diabetes, with the prerequisite that their expression profiles can be confirmed to be similar to the beta-cells in human islets of Langerhans, as indicated in a recent study by Jin et al. [13].

\section{Conclusions}

We conclude that the choice of method for extraction of membrane proteins from human islets of Langerhans will influence the number and identity of proteins detected in the proteomic analysis. Combining the results from varying extraction procedures enhanced the total number of membrane proteins identified, since every extraction method used contributed with uniquely identified membrane proteins. However, the hydrophobic phase of Triton X-114 phase separation was the extraction method yielding both the highest number and the highest proportion of membrane proteins. The list of proteins presented here contributes to the characterization of the human islet membrane proteome, which can be used as a reference in further studies and clinical applications in the diabetic research area.

Acknowledgment This study was supported by VINNOVA. We would like to thank the Proteomics Core Facility at the Sahlgrenska Academy, University of Gothenburg, which was funded by a grant from the Knut and Alice Wallenberg Foundation, as well as Nanoxis (Gothenburg, Sweden) for performing the LPI flow cell procedure.

\section{References}

1. Orci L, Unger RH. Functional subdivision of islets of Langerhans and possible role of D cells. Lancet. 1975;2:1243-4.

2. Stefan Y, Grasso S, Perrelet A, Orci L. A quantitative immunofluorescent study of the endocrine cell populations in the developing human pancreas. Diabetes. 1983;32:293-301.

3. Donath MY, Halban PA. Decreased beta-cell mass in diabetes: significance, mechanisms and therapeutic implications. Diabetologia. 2004:47:581-9.

4. Gepts W, Lecompte PM. The pancreatic islets in diabetes. Am J Med. 1981;70:105-15.

5. Butler AE, Janson J, Bonner-Weir S, Ritzel R, Rizza RA, Butler PC. Beta-cell deficit and increased beta-cell apoptosis in humans with type 2 diabetes. Diabetes. 2003;52:102-10.

6. Dubois M, Vacher P, Roger B, et al. Glucotoxicity inhibits late steps of insulin exocytosis. Endocrinology. 2007;148:1605-14.

7. Brunner Y, Schvartz D, Priego-Capote F, Coute Y, Sanchez JC. Glucotoxicity and pancreatic proteomics. J Proteomics. 2009;71:576-91.

8. Ahmed M. Proteomics and islet research. Adv Exp Med Biol. 2010;654:363-90.

9. Brunner Y, Coute Y, Iezzi M, et al. Proteomics analysis of insulin secretory granules. Mol Cell Proteomics. 2007;6:1007-17.

10. Dowling P, O'Driscoll L, O'Sullivan F, et al. Proteomic screening of glucose-responsive and glucose non-responsive MIN-6 beta cells reveals differential expression of proteins involved in protein folding, secretion and oxidative stress. Proteomics. 2006;6:6578-87.

11. D'Hertog W, Overbergh L, Lage K, et al. Proteomics analysis of cytokine-induced dysfunction and death in insulin-producing INS1E cells: new insights into the pathways involved. Mol Cell Proteomics. 2007;6:2180-99.

12. Hickey AJ, Bradley JW, Skea GL, et al. Proteins associated with immunopurified granules from a model pancreatic islet beta-cell system: proteomic snapshot of an endocrine secretory granule. J Proteome Res. 2009;8:178-86. 
13. Jin J, Park J, Kim K, et al. Detection of differential proteomes of human beta-cells during islet-like differentiation using iTRAQ labeling. J Proteome Res. 2009;8:1393-403.

14. Ahmed M, Bergsten P. Glucose-induced changes of multiple mouse islet proteins analysed by two-dimensional gel electrophoresis and mass spectrometry. Diabetologia. 2005;48:477-85.

15. Petyuk VA, Qian WJ, Hinault C, et al. Characterization of the mouse pancreatic islet proteome and comparative analysis with other mouse tissues. J Proteome Res. 2008;7:3114-26.

16. Boonen K, Baggerman G, D'Hertog W, et al. Neuropeptides of the islets of Langerhans: a peptidomics study. Gen Comp Endocrinol. 2007;152:231-41.

17. Sanchez JC, Chiappe D, Converset V, et al. The mouse SWISS2D PAGE database: a tool for proteomics study of diabetes and obesity. Proteomics. 2001;1:136-63.

18. Waanders LF, Chwalek K, Monetti M, Kumar C, Lammert E, Mann M. Quantitative proteomic analysis of single pancreatic islets. Proc Natl Acad Sci USA. 2009;106:18902-7.

19. Van Lommel L, Moreau Y, Pipeleers D, Jonas J-C, Schuit F. mRNA profiling of pancreatic beta cells: investigating mechanisms of diabetes. In: Hofmann W-K, editor. Gene expression profiling by microarrays: clinical implications. Cambridge: Cambridge University Press; 2006. p. 187-211.

20. Pan C, Kumar C, Bohl S, Klingmueller U, Mann M. Comparative proteomic phenotyping of cell lines and primary cells to assess preservation of cell type-specific functions. Mol Cell Proteomics. 2009;8:443-50.

21. Ahmed M, Forsberg J, Bergsten P. Protein profiling of human pancreatic islets by two-dimensional gel electrophoresis and mass spectrometry. J Proteome Res. 2005;4:931-40.

22. Metz TO, Jacobs JM, Gritsenko MA, et al. Characterization of the human pancreatic islet proteome by two-dimensional LC/MS/MS. J Proteome Res. 2006;5:3345-54.

23. Wu CC, Yates 3rd JR. The application of mass spectrometry to membrane proteomics. Nat Biotechnol. 2003;21:262-7.

24. Goto M, Eich TM, Felldin M, et al. Refinement of the automated method for human islet isolation and presentation of a closed system for in vitro islet culture. Transplantation. 2004;78:1367-75.

25. Bordier C. Phase separation of integral membrane proteins in Triton X-114 solution. J Biol Chem. 1981;256:1604-7.

26. Shevchenko A, Wilm M, Vorm O, Mann M. Mass spectrometric sequencing of proteins silver-stained polyacrylamide gels. Anal Chem. 1996;68:850-8.

27. Carlsohn E, Nystrom J, Karlsson H, Svennerholm AM, Nilsson CL. Characterization of the outer membrane protein profile from diseaserelated Helicobacter pylori isolates by subcellular fractionation and nano-LC FT-ICR MS analysis. J Proteome Res. 2006;5:3197-204.

28. Keller A, Nesvizhskii AI, Kolker E, Aebersold R. Empirical statistical model to estimate the accuracy of peptide identifications made by MS/ MS and database search. Anal Chem. 2002;74:5383-92.

29. Nesvizhskii AI, Keller A, Kolker E, Aebersold R. A statistical model for identifying proteins by tandem mass spectrometry. Anal Chem. 2003;75:4646-58.
30. Overington JP, Al-Lazikani B, Hopkins AL. How many drug targets are there? Nat Rev Drug Discov. 2006;5:993-6.

31. Ahmed FE. Sample preparation and fractionation for proteome analysis and cancer biomarker discovery by mass spectrometry. J Sep Sci. 2009;32:771-98.

32. Iliaki E, Poulaki V, Mitsiades N, Mitsiades CS, Miller JW, Gragoudas ES. Role of alpha 4 integrin (CD49d) in the pathogenesis of diabetic retinopathy. Invest Ophthalmol Vis Sci. 2009;50:4898-904.

33. Roth T, Podesta F, Stepp MA, Boeri D, Lorenzi M. Integrin overexpression induced by high glucose and by human diabetes: potential pathway to cell dysfunction in diabetic microangiopathy. Proc Natl Acad Sci USA. 1993;90:9640-4.

34. Kostidou E, Koliakos G, Kaloyianni M. Increased monocyte alphaL, alphaM and beta2 integrin subunits in diabetes mellitus. Clin Biochem. 2009;42:634-40.

35. Hemler ME. Targeting of tetraspanin proteins - potential benefits and strategies. Nat Rev Drug Discov. 2008;7:747-58.

36. Nyblom HK, Bugliani M, Fung E, et al. Apoptotic, regenerative, and immune-related signaling in human islets from type 2 diabetes individuals. J Proteome Res. 2009;8:5650-6.

37. Gesierich S, Paret C, Hildebrand D, et al. Colocalization of the tetraspanins, CO-029 and CD151, with integrins in human pancreatic adenocarcinoma: impact on cell motility. Clin Cancer Res. 2005;11:2840-52.

38. Grarup N, Andersen G, Krarup NT, et al. Association testing of novel type 2 diabetes risk alleles in the JAZF1, CDC123/CAMK1D, TSPAN8, THADA, ADAMTS9, and NOTCH2 loci with insulin release, insulin sensitivity, and obesity in a population-based sample of 4,516 glucose-tolerant middle-aged Danes. Diabetes. 2008;57:2534 40.

39. Ludvigsen E, Olsson R, Stridsberg M, Janson ET, Sandler S. Expression and distribution of somatostatin receptor subtypes in the pancreatic islets of mice and rats. J Histochem Cytochem. 2004;52:391-400.

40. Miettinen P, Ormio P, Hakonen E, Banerjee M, Otonkoski T. EGF receptor in pancreatic beta-cell mass regulation. Biochem Soc Trans. 2008;36:280-5.

41. Tyers M, Mann M. From genomics to proteomics. Nature. $2003 ; 422: 193-7$

42. Lindskog C, Asplund A, Engkvist M, Uhlen M, Korsgren O, Ponten F. Antibody-based proteomics for discovery and exploration of proteins expressed in pancreatic islets. Discov Med. 2010;9:565-78.

43. Uhlen M, Ponten F. Antibody-based proteomics for human tissue profiling. Mol Cell Proteomics. 2005;4:384-93.

44. Kersey PJ, Duarte J, Williams A, Karavidopoulou Y, Birney E, Apweiler R. The international protein index: an integrated database for proteomics experiments. Proteomics. 2004;4:19858 .

45. Narushima M, Kobayashi N, Okitsu T, et al. A human beta-cell line for transplantation therapy to control type 1 diabetes. Nat Biotechnol. 2005;23:1274-82. 